\title{
Convergence of generalized entropy minimizers in sequences of convex problems
}

\author{
Imre Csiszár \\ A. Rényi Institute of Mathematics \\ Hungarian Academy of Sciences \\ H-1364 Budapest, P.O.Box 127, Hungary \\ Email: csiszar@renyi.hu
}

\author{
František Matúš \\ Institute of Information Theory and Automation \\ Academy of Sciences of the Czech Republic \\ 18208 Prague, P.O.Box 18, Czech Republic \\ Email:matus@utia.cas.cz
}

\begin{abstract}
Integral functionals based on convex normal integrands are minimized over convex constraint sets. Generalized minimizers exist under a boundedness condition. Sequences of the minimization problems are studied when the constraint sets are nested. The corresponding sequences of generalized minimizers are related to the minimization over limit convex sets. Martingale theorems and moment problems are discussed.
\end{abstract}

\section{INTRODUCTION}

Let $(Z, \mathcal{Z}, \mu)$ be a $\sigma$-finite measure space. For real $\mathcal{Z}$-measurable functions $g$ on $Z$, let

$$
H_{\beta}: g \mapsto \int_{Z} \beta(z, g(z)) \mu(d z) .
$$

The functional $H_{\beta}$ is based on an integrand $\beta$ on $Z \times \mathbb{R}$ with values in $(-\infty,+\infty]$. Assumptions on $\beta$, summarized at the beginning of Section II, include strict convexity in the second coordinate, finiteness of $\beta(z, t)$ for $t>0$ and $\beta(z, t)=+\infty$ for $t<0$. Thus, $H_{\beta}$ is finite only when $g$ is nonnegative, $\mu$-a.e.

This work generalizes and strengthens existing results on minimization of the integral functional $H_{\beta}$ over convex sets $\mathcal{C}$ of functions $g$ for which $\inf _{\mathcal{C}} H_{\beta} \triangleq \inf _{g \in \mathcal{C}} H_{\beta}(g)$ is finite. If a minimizer $g$ in $\mathcal{C}$ exists, $H_{\beta}(g)=\inf _{\mathcal{C}} H_{\beta}$, then it is unique, $\mu$ a.e., by strict convexity. Otherwise, the minimizing sequences $g_{n}$ in $\mathcal{C}, H_{\beta}\left(g_{n}\right) \rightarrow \inf _{\mathcal{C}} H_{\beta}$, are of interest. The minimization of $H_{\beta}$ over $\mathcal{C}$ has a generalized minimizer if all minimizing sequences have a common limit, denoted here by $\hat{g}_{\mathcal{C}}$. Theorem 1 implies existence of $\hat{g}_{\mathcal{C}}$ when a single minimizing sequence is bounded. Corollary 1 presents equivalent assumptions.

For a sequence $\mathcal{C}_{n}$ of convex sets of functions $g$ let

$$
\left(\mathrm{P}_{n}\right) \quad J_{\beta}^{[n]}=\inf _{\mathcal{C}_{n}} H_{\beta}, \quad n \geqslant 1 .
$$

Assuming monotonicity, either $\mathcal{C}_{n} \supseteq \mathcal{C}_{n+1}$ or $\mathcal{C}_{n} \subseteq \mathcal{C}_{n+1}$, let $\mathcal{C}_{\infty}$ denote the intersection or union of the sets $\mathcal{C}_{n}$, respectively. The goal is to relate the problems $\left(\mathrm{P}_{n}\right)$ to

$\left(\mathrm{P}_{\infty}\right) \quad J_{\beta}^{[\infty]}=\inf _{\mathcal{C}_{\infty}} H_{\beta}$.

Theorems 2 and 3, formulated in Section III, deal with the limit behavior of the sequence $\hat{g}_{\mathcal{C}_{n}}$ of generalized minimizers in the problems $\left(\mathrm{P}_{n}\right)$. Under some conditions the convergence to the generalized minimizer $\hat{g}_{C_{\infty}}$ in $\left(\mathrm{P}_{\infty}\right)$ is established in Bregman distance based on $\beta$.

In Section IV, the results are applied to extend martingale theorems. Discussion of moment problems is presented in Section V. Proofs are postponed to Appendix.

\section{PRELIminaries AND ASSUMPTIONS}

It is assumed throughout that $\beta$ is a normal integrand [7, Chapter 14] such that $\beta(z, \cdot), z \in Z$, belongs to the class $\Gamma$ of functions $\gamma$ on $\mathbb{R}$ that are finite and strictly convex for $t>0$ and equal to $+\infty$ for $t<0$. The integrand is autonomous when $\gamma \in \Gamma$ exists such that $\beta(z, \cdot)=\gamma$ for all $z \in Z$.

A function $\gamma$ is asymptotically nonlinear if either the limit $\gamma^{\prime}(+\infty)$ of $\gamma^{\prime}(t)$ when $t \uparrow+\infty$ is infinite or the increasing function $t \mapsto t \gamma^{\prime}(+\infty)-\gamma(t)$ is unbounded. The integrand $\beta$ is asymptotically nonlinear if $\beta(z, \cdot)$ has the property for $\mu$-a.a. $z \in Z$.

From now on, all functions $f, g, h$ on $Z$ are assumed to be nonnegative and $\mathcal{Z}$-measurable. Equalities between them are considered $\mu$-a.e. If neither the positive nor the negative part of the function $z \mapsto \beta(z, g(z))$ is $\mu$-integrable, the integral of this function over $Z$ is $+\infty$ by convention.

The Bregman distance of $g$ and $h$, based on $\beta$, is given by

$$
B_{\beta}(g, h) \triangleq \int_{Z} \Delta_{\beta}(z, g(z), h(z)) \mu(d z)
$$

where $\Delta_{\beta}$ is a nonnegative integrand on $Z \times \mathbb{R}^{2}$ such that $\Delta_{\beta}(z, s, t)$ for $z \in Z$ and $s, t \geqslant 0$ is equal to

$$
\gamma(s)-\gamma(t)-\gamma_{\operatorname{sgn}(s-t)}^{\prime}(t)[s-t] \quad \text { if } \gamma_{+}^{\prime}(t) \text { is finite, }
$$

and $s \cdot(+\infty)$ otherwise. Here, $\gamma$ abbreviates $\beta(z, \cdot), \operatorname{sgn}(r)$ denotes + if $r \geqslant 0$ and - if $r<0$, and $\gamma_{ \pm}^{\prime}$ are the one-sided derivatives. The Bregman distance is nonnegative and vanishes if and only if $g=h$. For more technical details see [5].

A sequence of functions $g_{n}$ on $Z$ converges locally in the measure $\mu$ to a function $h$, in symbols $g_{n} \rightsquigarrow h$, if it converges in measure on each set $C$ of finite $\mu$-measure, thus

$$
\mu\left(C \cap\left\{\left|g_{n}-h\right|>\varepsilon\right\}\right) \rightarrow 0, \quad \varepsilon>0 .
$$

Either of the convergences $B_{\beta}\left(g_{n}, h\right) \rightarrow 0$ or $B_{\beta}\left(h, g_{n}\right) \rightarrow 0$ implies $g_{n} \rightsquigarrow h$ [5, Corollary 2.14].

A set of functions $\mathcal{H}$ on $Z$ is bounded locally in the measure $\mu$ if for each set $C \in \mathcal{Z}$ with finite $\mu(C)$ to every $\varepsilon>0$ there exists $K$ such that

$$
\sup _{h \in \mathcal{H}} \mu(C \cap\{h>K\}) \leqslant \varepsilon .
$$

A set of functions $\mathcal{G}$ or $\mathcal{H}$ is $\beta$-bounded or reversely $\beta$-bounded if there exist functions $g, h$ such that, respectively,

$$
\sup _{\mathcal{G}} B_{\beta}(\cdot, h)<+\infty \quad \text { or } \sup _{\mathcal{H}} B_{\beta}(g, \cdot)<+\infty .
$$




\section{MAIN RESULTS}

The following assertion extends [3, Theorem 1(c)] that is confined to autonomous integrands. There, an assumption of boundedness was missing, though implicitly used in a proof, see also [5, Example 10.5].

Theorem 1. Let $\mathcal{C}$ be a convex set of functions on $Z$ with finite $\inf _{\mathcal{C}} H_{\beta}$. If a minimizing sequence $g_{n} \in \mathcal{C}$ is bounded locally in measure then there exists a unique function $\hat{g}_{\mathcal{C}}$ such that

$$
H_{\beta}(g) \geqslant \inf _{\mathcal{C}} H_{\beta}+B_{\beta}\left(g, \hat{g}_{\mathcal{C}}\right), \quad g \in \mathcal{C} .
$$

Remark 1. If the finite infimum in (1) is attained, the function $\hat{g}_{\mathcal{C}}$ from Theorem 1 equals the minimizer and

$$
H_{\beta}(g) \geqslant H_{\beta}\left(\hat{g}_{\mathcal{C}}\right)+B_{\beta}\left(g, \hat{g}_{\mathcal{C}}\right), \quad g \in \mathcal{C} .
$$

Otherwise, $\hat{g}_{\mathcal{C}}$ is the generalized minimizer in the sense that $B_{\beta}\left(g_{n}, \hat{g}_{\mathcal{C}}\right) \rightarrow 0$ for every minimizing sequence $g_{n}$ in $\mathcal{C}$.

The assumption of boundedness has equivalent reformulations.

Corollary 1. Assuming $\inf _{\mathcal{C}} H_{\beta}$ is finite, the following assertions are equivalent.

(i) A minimizing sequence is bounded locally in measure.

(ii) Every minimizing sequence is eventually $\beta$-bounded.

(iii) The generalized minimizer exists.

(iv) The level sets of $H_{\beta}$ intersected with $\mathcal{C}$ are $\beta$-bounded.

Let $\mathcal{C}_{n}$ be a sequence of convex sets of nonnegative functions and $\left(\mathrm{P}_{n}\right)$ the corresponding minimization problems with values $J_{\beta}^{[n]}$. When nonincreasing/nondecreasing, in symbols $\mathcal{C}_{n} \searrow / \mathcal{C}_{n} \nearrow$, the intersection/union $\mathcal{C}_{\infty}$ gives rise to the problem $\left(\mathrm{P}_{\infty}\right)$. Correspondingly, the sequence of values $J_{\beta}^{[n]}$ is nondecreasing/nondecreasing and upper/lower bounded by $J_{\beta}^{[\infty]}$.

Theorem 2. Let $\mathcal{C}_{n} \searrow$ be a sequence of convex sets such that $J_{\beta}^{[n]}$ is finite. Let $H_{\beta}$ have a minimizing sequence in $\mathcal{C}_{1}$ that is bounded locally in measure. If the sequence $J_{\beta}^{[n]}$ has a finite limit then there exists a unique function $h_{\infty}$ such that $B_{\beta}\left(h_{\infty}, \hat{g}_{\mathcal{C}_{n}}\right) \rightarrow 0$ and

$$
H_{\beta}(g) \geqslant \lim _{n \rightarrow \infty} J_{\beta}^{[n]}+B_{\beta}\left(g, h_{\infty}\right), \quad g \in \mathcal{C}_{\infty} .
$$

If the finite limit of $J_{\beta}^{[n]}$ equals $J_{\beta}^{[\infty]}$ then $h_{\infty}=\hat{g}_{\mathcal{C}_{\infty}}$.

Remark 2. In Theorem 2, let the infima in the problems $\left(\mathrm{P}_{n}\right)$ be attained. By Remark $1, \hat{g}_{\mathcal{C}_{n}}$ are the unique minimizers. If the sequence $J_{\beta}^{[n]}$ is bounded then a unique function $h_{\infty}$ exists such that $\hat{g}_{\mathcal{C}_{n}}$ converges in the sense $B_{\beta}\left(h_{\infty}, \hat{g}_{\mathcal{C}_{n}}\right) \rightarrow 0$ and

$$
H_{\beta}(g) \geqslant \lim _{n \rightarrow \infty} H_{\beta}\left(\hat{g}_{\mathcal{C}_{n}}\right)+B_{\beta}\left(g, h_{\infty}\right), \quad g \in \mathcal{C}_{\infty} .
$$

By Lemma 4 in Appendix, $\hat{g}_{\mathcal{C}_{n}} \rightsquigarrow h_{\infty}$. Hence, along a subsequence $\hat{g}_{\mathcal{C}_{n}} \rightarrow h_{\infty}$, $\mu$-a.e. Often $H_{\beta}$ is lower semicontinuous, $\liminf _{n \rightarrow \infty} H_{\beta}\left(g_{n}\right) \geqslant H_{\beta}(g)$ whenever $g_{n} \rightarrow g$, $\mu$-a.e. Then,

$$
J_{\beta}^{[\infty]} \geqslant \lim _{n \rightarrow \infty} H_{\beta}\left(\hat{g}_{\mathcal{C}_{n}}\right) \geqslant H_{\beta}\left(h_{\infty}\right) .
$$

If $h_{\infty} \in \mathcal{C}_{\infty}$ then the inequalities are tight by (3), $h_{\infty}=\hat{g}_{\mathcal{C}_{\infty}}$ is the minimizer in the problem $\left(\mathrm{P}_{\infty}\right), B_{\beta}\left(\hat{g}_{\mathcal{C}_{\infty}}, \hat{g}_{\mathcal{C}_{n}}\right) \rightarrow 0$ and $H_{\beta}\left(\hat{g}_{\mathcal{C}_{n}}\right) \rightarrow H_{\beta}\left(\hat{g}_{\mathcal{C}_{\infty}}\right)$.
Example 1. Let $\mu$ be the measure on $\mathbb{R}^{2}$ expressible as sum of the probability measure (pm) sitting in the point $(0,1)$, the pm sitting in $(0,2)$ and the pm on $(0,+\infty)^{2}$ with the density $\left(x_{1}, x_{2}\right) \mapsto e^{-x_{1}-x_{2}}$, see [4, Example 1]. The functional $H_{\gamma}$ is based on the autonomous integrand $\gamma: t \mapsto t \ln t$.

Given $s \geqslant 1$, the functional $H_{\gamma}$ is minimized over

$$
\mathcal{C}_{n}=\bigcup\left\{\mathcal{G}(\varepsilon, s): 0 \leqslant \varepsilon \leqslant \frac{1}{n}\right\}
$$

where $\mathcal{G}\left(a_{1}, a_{2}\right)$ for $\left(a_{1}, a_{2}\right) \in \mathbb{R}^{2}$ denotes the set of nonnegative functions $g$ that satisfy the three moment constraints

$$
\int_{\mathbb{R}^{2}}\left(1, z_{1}, z_{2}\right) g\left(z_{1}, z_{2}\right) \mu\left(d z_{1}, d z_{2}\right)=\left(1, a_{1}, a_{2}\right) .
$$

The minimum of $H_{\gamma}$ over $\mathcal{G}(\varepsilon, s), \varepsilon>0$, is attained uniquely at the function $f_{\varepsilon, s}:\left(z_{1}, z_{2}\right) \mapsto e^{\vartheta_{1}^{*} z_{1}+\vartheta_{2}^{*} z_{2}-\Lambda\left(\vartheta_{1}^{*}, \vartheta_{2}^{*}\right)}$ where

$$
\Lambda:\left(\vartheta_{1}, \vartheta_{2}\right) \mapsto \ln \left[e^{\vartheta_{2}}+e^{2 \vartheta_{2}}+\frac{1}{1-\vartheta_{1}} \frac{1}{1-\vartheta_{2}}\right], \quad \vartheta_{1}, \vartheta_{2}<1,
$$

is the log-Laplace transform of $\mu$ and $\left(\vartheta_{1}^{*}, \vartheta_{2}^{*}\right)$ is the unique solution of $\nabla \Lambda\left(\vartheta_{1}^{*}, \vartheta_{2}^{*}\right)=(\varepsilon, s)$. Thus,

$$
\begin{aligned}
& \frac{1}{\left(1-\vartheta_{1}^{*}\right)^{2}} \frac{1}{1-\vartheta_{2}^{*}}=\varepsilon\left[e^{\vartheta_{2}^{*}}+e^{2 \vartheta_{2}^{*}}+\frac{1}{1-\vartheta_{1}^{*}} \frac{1}{1-\vartheta_{2}^{*}}\right] \\
& e^{\vartheta_{2}^{*}}+2 e^{2 \vartheta_{2}^{*}}+\frac{1}{1-\vartheta_{1}^{*}} \frac{1}{\left(1-\vartheta_{2}^{*}\right)^{2}}=s\left[e^{\vartheta_{2}^{*}}+e^{2 \vartheta_{2}^{*}}+\frac{1}{1-\vartheta_{1}^{*}} \frac{1}{1-\vartheta_{2}^{*}}\right] .
\end{aligned}
$$

For fixed $s>s^{*} \triangleq \frac{1+2 e}{1+e}$, the second equation and $\vartheta_{2}^{*} \nearrow 1 \mathrm{imply}$

$$
\frac{1}{1-\vartheta_{1}^{*}} \frac{1}{\left(1-\vartheta_{2}^{*}\right)^{2}} \rightarrow\left(e+e^{2}\right)\left(s-s^{*}\right) \text { and } \vartheta_{1}^{*} \rightarrow-\infty .
$$

By the first equation, $\vartheta_{1}^{*} \varepsilon \rightarrow 0$ and $\varepsilon \rightarrow 0$. Hence, when $\varepsilon \downarrow 0$ the functions $f_{\varepsilon, s}$ converge to zero on $(0,+\infty)^{2}$, to $\frac{1}{1+e}$ at $(0,1)$ and to $\frac{e}{1+e}$ at $(0,2)$. Further,

$\min _{\mathcal{G}(\varepsilon, s)} H_{\gamma}=\vartheta_{1}^{*} \varepsilon+\vartheta_{2}^{*} s-\Lambda\left(\vartheta_{1}^{*}, \vartheta_{2}^{*}\right) \rightarrow s-1-\ln (1+e)$.

This minimum is actually $\Lambda^{*}(\varepsilon, s)$ where $\Lambda^{*}$ is the conjugate of $\Lambda$. By calculus, for $s>s^{*}$

$$
\begin{aligned}
\Lambda^{*}(\varepsilon, s) & \leqslant \sup _{\vartheta_{1}, \vartheta_{2}<1}\left[\vartheta_{1} \varepsilon+\vartheta_{2} s-\ln \left[e^{\vartheta_{2}}+e^{2 \vartheta_{2}}+\frac{1}{1-\vartheta_{1}} e^{\vartheta_{2}}\right]\right] \\
& =s-1+\sup _{\vartheta_{1}<1}\left[\vartheta_{1} \varepsilon-\ln \left[1+e+\frac{1}{1-\vartheta_{1}}\right]\right] \\
& =s-1-\ln (1+e)-2 \sqrt{\varepsilon /(1+e)}+O(\varepsilon) .
\end{aligned}
$$

The sets $\mathcal{C}_{n}$ intersect to $\mathcal{C}_{\infty}=\mathcal{G}(0, s)$. For $s \leqslant 2$ this family contains the single function $f_{0, s}$ that equals $2-s$ at $(0,1), s-1$ at $(0,2)$, and 0 otherwise. Hence,

$$
J_{\beta}^{[\infty]}=(s-1) \ln (s-1)+(2-s) \ln (2-s), \quad 1 \leqslant s \leqslant 2 .
$$

If $s>2$ then $\mathcal{G}(0, s)$ is empty, thus $J_{\beta}^{[\infty]}=+\infty$.

For $s>s^{*}$ it follows that the infimum in $J_{\beta}^{[n]}$ is attained and

$$
\lim _{n \rightarrow \infty} J_{\beta}^{[n]}=s-1-\ln (1+e)<J_{\beta}^{[\infty]} .
$$

Thus, the limit is finite but smaller than $J_{\beta}^{[\infty]}$, which is even infinite for $s>2$. When $n$ is sufficiently large the minimizer $\hat{g}_{\mathcal{C}_{n}}$ in the problem $\left(\mathrm{P}_{n}\right)$ is equal to the minimizer $f_{1 / n, s}$ of $H_{\gamma}$ over $\mathcal{G}(1 / n, s)$, using the upper bound on $\Lambda^{*}$. Theorem 2 applies. The function $h_{\infty}=f_{0, s^{*}}$ is different from $\hat{g}_{C_{\infty}}=f_{0, s}$ for $s^{*}<s \leqslant 2$, while $\hat{g}_{C_{\infty}}$ is not defined for $s>2$. 
Theorem 3. Let $\beta$ be asymptotically nonlinear and $\mathcal{C}_{n} \nearrow$ be a sequence of convex sets such that $J_{\beta}^{[n]}$ is finite. Let $H_{\beta}$ have a minimizing sequence in $\mathcal{C}_{n}$ that is bounded locally in measure, $n \geqslant 1$. If the sequence $J_{\beta}^{[n]}$ has a finite limit then the limit equals $J_{\beta}^{[\infty]}$, the minimizing sequences in $\mathcal{C}_{\infty}$ are $\beta$-bounded and $B_{\beta}\left(\hat{g}_{\mathcal{C}_{n}}, \hat{g}_{C_{\infty}}\right) \rightarrow 0$.

Example 2. Let $\mu$ be the Lebesgue measure on $[0,1]$. The functional $H_{\gamma}$ is based on $\gamma: t \mapsto t \ln t$ and is minimized over

$$
\mathcal{C}_{n}=\left\{g \geqslant 0: \int_{0}^{1} g d \mu=1, g(t)=2 t \text { for } t \leqslant \frac{1}{n}\right\} .
$$

The minimizer exists, $\hat{g}_{\mathcal{C}_{n}}(t)=1+\frac{1}{n}, t \geqslant \frac{1}{n}$, and

$$
J_{\beta}^{[n]}=\int_{0}^{1 / n} 2 t \ln (2 t) d t+\left(1-\frac{1}{n^{2}}\right) \ln \left(1+\frac{1}{n}\right) \searrow 0 .
$$

By Theorem $3, J_{\beta}^{[\infty]}=0$ and $\hat{g}_{C_{\infty}} \equiv 1$ is the generalized minimizer in the problem $\left(\mathrm{P}_{\infty}\right)$, which has however no minimizer because $\hat{g}_{C_{\infty}}$ is not in $\mathcal{C}_{\infty}=\bigcup_{n \geqslant 1} \mathcal{C}_{n}$. Instead, $\hat{g}_{C_{\infty}}$ is a minimizer when minimizing over all functions integrating to 1 .

\section{Martingale theorems}

In this section Theorems 2 and 3 are related to convergence of conditional expectations in Bregman distances.*

A function $f$ has the covering property w.r.t. a sub- $\sigma$-algebra $\mathcal{Y}$ of $\mathcal{Z}$ (and $\mu$ ) if $Z$ is the union of at most countably many sets $Y \in \mathcal{Y}$ with $\int_{Y} f d \mu$ finite. By Radon-Nikodym theorem, there exists a $\mathcal{Y}$-measurable function $f_{\mid \mathcal{Y}}=f_{\mid \mathcal{Y}, \mu}$ that satisfies $\int_{Y} f_{\mid \mathcal{Y}} d \mu=\int_{Y} f d \mu$ for $Y \in \mathcal{Y}$. It is nonnegative and unique. If $f>0$ then $f_{\mid \mathcal{Y}}>0$.

Let sub- $\sigma$-algebras $\mathcal{Y}_{n}$ of $\mathcal{Z}$ be nested as $\mathcal{Y}_{n} \subseteq \mathcal{Y}_{n+1}$, in symbols $\mathcal{Y}_{n} \nearrow$, and generate $\mathcal{Y}_{\infty}$. A martingale theorem asserts that if $f$ has the covering property w.r.t. $\mathcal{Y}_{1}$ then $f_{\mid \mathcal{Y}_{n}} \rightarrow f_{\mid \mathcal{Y}_{\infty}}$, $\mu$-a.e. In a backward martingale theorem, sub- $\sigma$-algebras are nested as $\mathcal{Y}_{n} \supseteq \mathcal{Y}_{n+1}$ and intersect to $\mathcal{Y}_{\infty}$.

Let $\mathcal{D}_{\mathcal{Y}, f}$ denote the set of nonnegative functions $g$ that satisfy $\int_{Y} g d \mu=\int_{Y} f d \mu$ for $Y \in \mathcal{Y}$. The minimization of the functionals $H_{\beta}$ over the convex set $\mathcal{C}=\mathcal{D}_{\mathcal{Y}, f}$ is not considered here in full generality but only for integrands

$$
\beta:(z, t) \mapsto h(z) \gamma\left(\frac{t}{h(z)}\right), \quad z \in Z, t \in \mathbb{R},
$$

where $\gamma \in \Gamma$ and $h$ is a positive function on $Z$. If $h \equiv 1$ then the integrand is autonomous, which can be arranged replacing $\mu$ by $\nu$ with $d \nu=h d \mu$ and $g$ by $g h$, see [5, Appendix C].

It is further assumed that $\gamma$ is differentiable, nonnegative and $\gamma(1)=0$. Then, $H_{\beta}(g)$ becomes the $\gamma$-divergence $D_{\gamma}(g, h)$ of a function $g$ from $h$

$$
H_{\beta}(g)=\int_{Z} h \gamma\left(\frac{g}{h}\right) d \mu=D_{\gamma}(g, h) .
$$

Lemma 1. If $f \geqslant 0$ and $h>0$ have the covering property w.r.t. a sub- $\sigma$-algebra $\mathcal{Y}$ of $\mathcal{Z}$ and $\mu$ then

$$
\min _{g \in \mathcal{D}_{\mathcal{Y}, f}} D_{\gamma}(g, h)=D_{\gamma}\left(f_{\mid \mathcal{Y}}, h_{\mid \mathcal{Y}}\right) .
$$

If finite, the minimum is attained at $g=f_{\mid \mathcal{Y}} h / h_{\mid \mathcal{Y}}$.

* Proofs of the assertions presented here are not included. They are available for reviewer's purposes at http://staff.utia.cas.cz/matus/
When the minimum in Lemma 1 is finite, Theorem 1 for $H_{\beta}=D_{\gamma}(\cdot, h)$ and $\mathcal{C}=\mathcal{D}_{\mathcal{Y}, f}$ features the minimizer $\hat{g}_{\mathcal{D}, f}$ equal to $f_{\mid \mathcal{Y}} h / h_{\mid \mathcal{Y}}$. Ineq. (1) is tight

$$
D_{\gamma}(g, h)=D_{\gamma}\left(f_{\mid \mathcal{Y}}, h_{\mid \mathcal{Y}}\right)+B_{\beta}\left(g, f_{\mid \mathcal{Y}} h / h_{\mid \mathcal{Y}}\right), \quad g \in \mathcal{D}_{\mathcal{Y}, f} .
$$

In fact, if the right hand side is finite the Bregman distance is

$$
\int_{Z}\left[h \gamma\left(\frac{g}{h}\right)-h \gamma\left(\frac{f_{\mid \mathcal{Y}}}{h_{\mid \mathcal{Y}}}\right)-\gamma^{\prime}\left(\frac{f_{\mid \mathcal{Y}}}{h_{\mid \mathcal{Y}}}\right)\left[g-\frac{f_{\mid \mathcal{Y}} h}{h_{\mid \mathcal{Y}}}\right]\right] d \mu .
$$

The third term can be omitted since $g_{\mid \mathcal{Y}}=f_{\mid \mathcal{Y}}$. For the autonomous integrand, $h \equiv 1, \hat{g}_{\mathcal{D}_{\mathcal{Y}, f}}=f_{\mid \mathcal{Y}}$ and (1) takes the form

$$
H_{\gamma}(g)=H_{\gamma}\left(f_{\mid \mathcal{Y}}\right)+B_{\gamma}\left(g, f_{\mid \mathcal{Y}}\right), \quad g \in \mathcal{D}_{\mathcal{Y}, f} .
$$

Lemma 2. If sub- $\sigma$-algebras $\mathcal{Y}_{n} \nearrow$, generate $\mathcal{Y}_{\infty}$, and $f \geqslant 0$ and $h>0$ have the covering property w.r.t. $\mathcal{Y}_{1}$ then

$$
D_{\gamma}\left(f_{\mid \mathcal{Y}_{n}}, h_{\mid \mathcal{Y}_{n}}\right) \nearrow D_{\gamma}\left(f_{\mid \mathcal{Y}_{\infty}}, h_{\mid \mathcal{Y}_{\infty}}\right) .
$$

Theorem 2 restricted to the autonomous integrands applies to sequences of sets of the type $\mathcal{D}_{\mathcal{Y}, f}$. Lemmas 1 and 2 are invoked in the proof.

Corollary 2. Under the assumptions of Lemma 2 with $h \equiv 1$, if $H_{\gamma}\left(f_{\mid \mathcal{Y}_{\infty}}\right)$ is finite then $B_{\gamma}\left(f_{\mid \mathcal{Y}_{\infty}}, f_{\mid \mathcal{Y}_{n}}\right) \rightarrow 0$.

The choice $\gamma: t \mapsto t \ln t-t+1$ gives the convergence of relative entropies in Lemma 2, see [6, Theorem 2], while the assertion of Corollary 2 seems to be new.

A consequence of Theorem 3 is analogous to Corollary 2 .

Corollary 3. Let $\gamma$ be asymptotically nonlinear. If $\mathcal{Y}_{n} \searrow$, intersect to $\mathcal{Y}_{\infty}, f \geqslant 0$ and $h>0$ have the covering property w.r.t. $\mathcal{Y}_{\infty}$, and $D_{\gamma}\left(f_{\mid \mathcal{Y}_{1}}, h_{\mid \mathcal{Y}_{1}}\right)$ is finite then

$$
D_{\gamma}\left(f_{\mid \mathcal{Y}_{n}}, h_{\mid \mathcal{Y}_{n}}\right) \searrow D_{\gamma}\left(f_{\mid \mathcal{Y}_{\infty}}, h_{\mid \mathcal{Y}_{\infty}}\right),
$$

and if $H_{\gamma}\left(f_{\mid \mathcal{Y}_{1}}\right)$ is finite then $B_{\gamma}\left(f_{\mid \mathcal{Y}_{n}}, f_{\mid \mathcal{Y}_{\infty}}\right) \rightarrow 0$.

Choosing $\gamma: t \mapsto t \ln t-t+1$ the convergence of relative entropies follows, see [6, Theorem 3].

\section{DISCUSSION}

The minimization of integral functionals studied in this contribution includes a number of special situations that appeared before. The relations to literature are discussed at length in [5, Section 11]. The focus here is on sequences of generalized minimizers for entropy-like functionals, which is of novelty even for the autonomous integrands. The generalization matters, see the simple situation in Example 2.

To make Theorem 2 operational, conditions for the convergence $J_{\beta}^{[n]} \rightarrow J_{\beta}^{[\infty]}<+\infty$ are desirable. By Remark 2 , it is sufficient to have $\beta \geqslant 0$, making $H_{\beta}$ lower semicontinuous, and $h_{\infty} \in \mathcal{C}_{\infty}$. An alternative is to assume that $\mu$ is finite, and $\beta$ bounded below. Likewise, for Theorem 3 boundedness of $J_{\beta}^{[n]}$ should be under control. For more general sequences of convex sets, see [2], [8].

In moment problems, $\mathcal{C}$ is the set of functions $g$ satisfying

$$
\int_{Z} \varphi_{j} g d \mu=a_{j}, \quad j=1, \ldots, d,
$$


where $\varphi_{j}: Z \rightarrow \mathbb{R}$ are given moment functions and $a_{j} \in \mathbb{R}$ are prescribed moments. Feasibility is resolved by the concept of conic core [5]. If $\inf _{\mathcal{C}} H_{\beta}$ is finite then the boundedness condition from Theorem 1 is equivalent to the modified dual constraint qualification. The generalized minimizer can be explicitly described avoiding primary constraint qualification [5].

A well-understood special limiting situation is when $\mu$ is finite, $H_{\gamma}$ is based on $\gamma \in \Gamma$ with $\gamma(t) / t \rightarrow+\infty$,

$$
\mathcal{C}_{n}=\left\{g \in L_{1}(\mu): \int_{Z} \varphi_{j} g d \mu=a_{j}, j=1, \ldots, n\right\}
$$

where $\varphi_{j} \in L_{\infty}(\mu)$, and $\mathcal{C}_{\infty}=\bigcap_{n \geqslant 1} \mathcal{C}_{n}$ contains a function $g$ with $H_{\gamma}(g)$ finite. Then, $H_{\gamma}$ is bounded below, $\mathcal{C}_{n}$ are weakly closed, $H_{\gamma}$ has weakly compact level sets and is strongly lower semicontinuous in $L_{1}(\mu)$. Therefore, $\hat{g}_{\mathcal{C}_{n}}$ and $\hat{g}_{\mathcal{C}_{\infty}}$ are minimizers [8, Theorem 1]. The convergence $J_{\beta}^{[n]} \rightarrow J_{\beta}^{[\infty]}<+\infty$ takes place. By Theorem 2, $B_{\gamma}\left(\hat{g}_{\mathcal{C}_{\infty}}, \hat{g}_{\mathcal{C}_{n}}\right) \rightarrow 0$. If $\gamma(t)=t \ln t$ this improves the previously known convergence in $L_{1}(\mu)[1$, Corollary 3.3]. See also [3] for a sufficient condition on $\gamma$ to conclude this convergence.

\section{APPENDIX}

Any $\beta$-bounded set is bounded locally in measure and so is any reversely $\beta$-bounded set provided $\beta$ is asymptotically nonlinear, see Corollary 5.

The following appeared previously as [5, Lemma 2.13].

Lemma 3. To any set $C \in \mathcal{Z}$ of finite $\mu$-measure and $K, \xi, \varepsilon$ positive there exists $\delta>0$ such that for functions $g$ and $h$ either of $B_{\beta}(g, h) \leqslant \delta$ or $B_{\beta}(h, g) \leqslant \delta$ implies

$$
\mu(C \cap\{|g-h|>\varepsilon\})<\xi+\mu(C \cap\{g>K\}) .
$$

Lemma 4. If a sequence $g_{n}$ is bounded locally in measure, $h_{n, m}$ is an array of functions and $B_{\beta}\left(g_{n}, h_{n, m}\right) \rightarrow 0$ with $n, m \rightarrow \infty(n \geqslant m$ or $m \geqslant n)$ then $g_{n}-h_{n, m} \rightsquigarrow 0$ with $n, m \rightarrow \infty(n \geqslant m$ or $m \geqslant n)$.

Proof: Since the sequence is bounded, if $C \in \mathcal{Z}$ has finite $\mu$-measure and $\xi>0$ then there exists $K>0$ such that $\mu\left(C \cap\left\{g_{n}>K\right\}\right)<\xi$ for all $n \geqslant 1$. By Lemma 3, for $\varepsilon>0$ there exists $\delta>0$ such that if $B_{\beta}\left(g_{n}, h_{n, m}\right) \leqslant \delta$ then

$$
\mu\left(C \cap\left\{\left|h_{n, m}-g_{n}\right|>\varepsilon\right\}\right)<\xi+\mu\left(C \cap\left\{g_{n}>K\right\}\right)<2 \xi .
$$

The assumption on convergence implies that this holds eventually in $n, m$. Hence, the local convergence follows.

Corollary 4. If a sequence $g_{n}$ is bounded locally in measure and $B_{\beta}\left(g_{n}, g_{m}\right) \rightarrow 0$ with $n \geqslant m \rightarrow \infty$ or $m \geqslant n \rightarrow \infty$ then $g_{n}$ converges locally in measure.

Proof: Lemma 4 is applied with $h_{n, m}=g_{m}$ to conclude that $g_{n}-g_{m} \rightsquigarrow 0$ with $n, m$ as above. In either case, $g_{n}$ is Cauchy locally in measure, and the convergence follows.

Proof of Theorem 1: The main ingredient is the identity involving integral functionals and Bregman distances

$$
\begin{aligned}
& t H_{\beta}\left(g_{1}\right)+(1-t) H_{\beta}\left(g_{2}\right)-H_{\beta}\left(t g_{1}+(1-t) g_{2}\right) \\
& \quad=t B_{\beta}\left(g_{1}, h\right)+(1-t) B_{\beta}\left(g_{2}, h\right)-B_{\beta}\left(t g_{1}+(1-t) g_{2}, h\right)
\end{aligned}
$$

where $g_{1}, g_{2}$ and $h$ are nonnegative functions, $0<t<1$ and the $H_{\beta}$-terms and $B_{\beta}\left(t g_{1}+(1-t) g_{2}, h\right)$ are finite.

By assumption, there exists a minimizing sequence $g_{n}$ in $\mathcal{C}$ that is bounded locally in measure and finite $H_{\beta}\left(g_{n}\right)$ converge to finite $\inf _{\mathcal{C}} H_{\beta}$. Then, the identity implies

$$
\begin{aligned}
\frac{1}{2} H_{\beta}\left(g_{n}\right) & +\frac{1}{2} H_{\beta}\left(g_{m}\right)-H_{\beta}\left(h_{n, m}\right) \\
& =\frac{1}{2} B_{\beta}\left(g_{n}, h_{n, m}\right)+\frac{1}{2} B_{\beta}\left(g_{m}, h_{n, m}\right)
\end{aligned}
$$

where $h_{n, m}=\frac{1}{2}\left(g_{n}+g_{m}\right)$. The left-hand side tends to 0 with $n, m \rightarrow \infty$ as $\boldsymbol{H}_{\beta}\left(h_{n, m}\right)$ converges to $\inf _{\mathcal{C}} \boldsymbol{H}_{\beta}$ due to convexity of $H_{\beta}$. Then, $B_{\beta}\left(g_{n}, h_{n, m}\right) \rightarrow 0$ and $B_{\beta}\left(g_{m}, h_{n, m}\right) \rightarrow 0$. By Lemma $4, g_{n}-h_{n, m} \rightsquigarrow 0$ and $g_{m}-h_{n, m} \rightsquigarrow 0$. Hence, $g_{n}-g_{m} \rightsquigarrow 0$ which expresses that the sequence $g_{n}$ is Cauchy locally in measure. Going to a subsequence if necessary, $g_{n} \rightarrow \hat{g}_{\mathcal{C}}, \mu$-a.e., for some function $\hat{g}_{\mathcal{C}}$.

Taking $g \in \mathcal{C}$ with finite $H_{\beta}(g)$ and $h_{n}=t_{n} g+\left(1-t_{n}\right) g_{n}$ with $0<t_{n}<1$, the identity implies

$$
\begin{aligned}
t_{n} H_{\beta}(g) & +\left(1-t_{n}\right) H_{\beta}\left(g_{n}\right)-H_{\beta}\left(h_{n}\right) \\
& =t_{n} B_{\beta}\left(g, h_{n}\right)+\left(1-t_{n}\right) B_{\beta}\left(g_{n}, h_{n}\right) .
\end{aligned}
$$

If $t_{n} \downarrow 0$ then $H_{\beta}\left(h_{n}\right) \rightarrow \inf _{\mathcal{C}} H_{\beta}$. Hence, $t_{n}$ can decrease slowly enough to make $\frac{1}{t_{n}}\left[H_{\beta}\left(g_{n}\right)-H_{\beta}\left(h_{n}\right)\right]$ converging to zero. Dividing by $t_{n}$,

$$
H_{\beta}(g)-\inf _{\mathcal{C}} H_{\beta} \geqslant \limsup _{n \rightarrow \infty}\left[\frac{H_{\beta}\left(h_{n}\right)-H_{\beta}\left(g_{n}\right)}{t_{n}}+B_{\beta}\left(g, h_{n}\right)\right] .
$$

Since $h_{n} \rightarrow \hat{g}_{\mathcal{C}}, \mu$-a.e., ineq. (1) follows by lower semicontinuity of the Bregman distance [5, Lemma 2.12].

Proof of Theorem 2: By assumptions, Theorem 1 applied to $\mathcal{C}_{1}$ and Corollary 1 , the level sets of $H_{\beta}$ intersected with $\mathcal{C}_{1}$ are $\beta$-bounded. Then, Theorem 1 and Corollary 1 apply to all $\mathcal{C}_{n}$ and the generalized minimizers $\hat{g}_{\mathcal{C}_{n}}$ exist. For $n \geqslant m$, ineq. (1) and $\mathcal{C}_{n} \subseteq \mathcal{C}_{m}$ imply

$$
H_{\beta}(g) \geqslant J_{\beta}^{[m]}+B_{\beta}\left(g, \hat{g}_{\mathcal{C}_{m}}\right), \quad g \in \mathcal{C}_{n} .
$$

If $g_{k} \in \mathcal{C}_{n}$ is a minimizing sequence in the problem $\left(\mathrm{P}_{n}\right)$ then (4) with $g_{k}$ instead of $g$ is limited in $k \rightarrow \infty$ and gives

$$
\text { (5) } J_{\beta}^{[n]} \geqslant J_{\beta}^{[m]}+\limsup _{k \rightarrow \infty} B_{\beta}\left(g_{k}, \hat{g}_{\mathcal{C}_{m}}\right) \geqslant J_{\beta}^{[m]}+B_{\beta}\left(\hat{g}_{\mathcal{C}_{n}}, \hat{g}_{\mathcal{C}_{m}}\right)
$$

due to $g_{k} \rightsquigarrow \hat{g}_{\mathcal{C}_{n}}$ and lower semicontinuity of the Bregman distance [5, Lemma 2.12]. Hence, $B_{\beta}\left(\hat{g}_{\mathcal{C}_{n}}, \hat{g}_{\mathcal{C}_{m}}\right) \rightarrow 0$ when $n, m \rightarrow \infty$ and $n \geqslant m$. In addition, $\hat{g}_{\mathcal{C}_{n}}$ is $\beta$-bounded as

$$
\lim _{n \rightarrow \infty} J_{\beta}^{[n]}-J_{\beta}^{[1]} \geqslant \sup _{n} B_{\beta}\left(\hat{g}_{\mathcal{C}_{n}}, \hat{g}_{\mathcal{C}_{1}}\right) .
$$

By Corollary $4, \hat{g}_{\mathcal{C}_{n}} \rightsquigarrow h_{\infty}$ for a function $h_{\infty}$. Limiting in (5) with $n \rightarrow \infty$ and lower semicontinuity imply

$$
\lim _{n \rightarrow \infty} J_{\beta}^{[n]} \geqslant J_{\beta}^{[m]}+B_{\beta}\left(h_{\infty}, \hat{g}_{\mathcal{C}_{m}}\right), \quad m \geqslant 1 .
$$

Hence, $B_{\beta}\left(h_{\infty}, \hat{g}_{\mathcal{C}_{m}}\right) \rightarrow 0$ with $m \rightarrow \infty$. It follows from (4) restricted to $\mathcal{C}_{\infty} \subseteq \mathcal{C}_{n}$ that

$$
H_{\beta}(g) \geqslant \lim _{m \rightarrow \infty} J_{\beta}^{[m]}+\limsup _{m \rightarrow \infty} B_{\beta}\left(g, \hat{g}_{\mathcal{C}_{m}}\right), \quad g \in \mathcal{C}_{\infty} .
$$

This, $\hat{g}_{\mathcal{C}_{m}} \rightsquigarrow h_{\infty}$ and lower semicontinuity imply ineq. (2). 
When the value $J_{\beta}^{[\infty]}$ in $\left(\mathrm{P}_{\infty}\right)$ is finite then ineq. (2) implies that the level sets of $H_{\beta}$ intersected with $\mathcal{C}_{\infty}$ are $\beta$-bounded. Theorem 1 together with Corollary 1 imply existence of a function $\hat{g}_{C_{\infty}}$ such that

$$
H_{\beta}(g) \geqslant J_{\beta}^{[\infty]}+B_{\beta}\left(g, \hat{g}_{C_{\infty}}\right), \quad g \in \mathcal{C}_{\infty} .
$$

When $\lim _{n \rightarrow \infty} J_{\beta}^{[n]}=J_{\beta}^{[\infty]}$, ineqs. (6) and (2) differ only in $h_{\infty}$ and $\hat{g}_{C_{\infty}}$. Then, the two functions coincide due to the uniqueness stated in Theorem 1.

Remark 3. In Theorem 2, if $g_{n} \in \mathcal{C}_{n}$ and $H_{\beta}\left(g_{n}\right)-J_{\beta}^{[n]} \rightarrow 0$ then $g_{n} \rightsquigarrow h_{\infty}$. In fact, ineq. (4) implies

$$
\lim _{n \rightarrow \infty} J_{\beta}^{[n]} \geqslant J_{\beta}^{[m]}+\limsup _{n \rightarrow \infty} B_{\beta}\left(g_{n}, \hat{g}_{\mathcal{C}_{m}}\right) .
$$

Then, $g_{n}$ is eventually $\beta$-bounded. Since $B_{\beta}\left(g_{n}, g_{\mathcal{C}_{n}}\right) \rightarrow 0$, due to ineq. (4) with $n=m$, it follows that $g_{n}-g_{\mathcal{C}_{n}} \rightsquigarrow 0$ by Lemma 4. This and $g_{n} \rightsquigarrow \hat{g}_{C_{\infty}}$ imply $g_{n} \rightsquigarrow h_{\infty}$.

The following is an extended version of [5, Lemma 9.9 $]^{\dagger}$.

Lemma 5. Let $C \in \mathcal{Z}$ have finite $\mu$-measure and $L, \xi, \delta>0$. There exists $K>L$ such that if $B_{\beta}(g, h) \leqslant \delta$ for functions $g, h$ then

$$
\mu(C \cap\{g>K\})<\xi+\mu(C \cap\{h>L\}) .
$$

The assertion holds also assuming instead $B_{\beta}(h, g) \leqslant \delta$ and the asymptotic nonlinearity of $\beta$.

Proof: Let $M=2 \delta / \xi$. By monotonicity, for $K>L$

$$
\begin{aligned}
B_{\beta}(g, h) & \geqslant \int_{\{g>K, h \leqslant L\}} \Delta_{\beta}(z, g(z), h(z)) \mu(d z) \\
& \geqslant \int_{\{g>K, h \leqslant L\}} \Delta_{\beta}(z, K, L) \mu(d z) \\
& \geqslant M \cdot \mu\left(C \cap\left\{\Delta_{\beta}(\cdot, K, L) \geqslant M, g>K, h \leqslant L\right\}\right) .
\end{aligned}
$$

Then, $\mu(C \cap\{g>K\})$ is at most

$\frac{1}{M} B_{\beta}(g, h)+\mu\left(C \cap\left\{\Delta_{\beta}(\cdot, K, L)<M\right\}\right)+\mu(C \cap\{h>L\})$.

If $B_{\beta}(g, h) \leqslant \delta$ then $\frac{1}{M} B_{\beta}(h, g) \leqslant \frac{1}{2} \xi$. By strict convexity, $\Delta_{\beta}(z, K, L)$ increases to $+\infty$ when $K \uparrow+\infty$. Therefore, for $K>L$ sufficiently large $\mu\left(C \cap\left\{\Delta_{\beta}(\cdot, K, L)<M\right\}\right)<\frac{1}{2} \xi$, and the assertion follows.

If $B_{\beta}(h, g) \leqslant \delta$ then for $K>L$

$$
\begin{aligned}
\delta & \geqslant \int_{\{g>K, h \leqslant L\}} \Delta_{\beta}(z, h(z), g(z)) \mu(d z) \\
& \geqslant \int_{\{g>K, h \leqslant L\}} \Delta_{\beta}(z, L, K) \mu(d z) \\
& \geqslant M \cdot \mu\left(C \cap\left\{\Delta_{\beta}(\cdot, L, K) \geqslant M, g>K, h \leqslant L\right\}\right) .
\end{aligned}
$$

Therefore, $\mu(C \cap\{g>K\})$ is at most

$$
\frac{1}{2} \xi+\mu\left(C \cap\left\{\Delta_{\beta}(\cdot, L, K)<M\right\}\right)+\mu(C \cap\{h>L\}) .
$$

To conclude $\Delta_{\beta}(z, L, K) \uparrow+\infty$ with $K \uparrow+\infty$, the asymptotic nonlinearity of $\beta(z, \cdot)$ suffices. The modified assertion follows as above.

Corollary 5. If a set $\mathcal{G}$ of functions is $\beta$-bounded then is bounded locally in measure. The conclusion holds also for

\footnotetext{
${ }^{\dagger}$ In Lemma 9.9 the arguments in $B_{\beta}(h, g)$ are erroneously interchanged.
}

sets $\mathcal{H}$ that are reversely $\beta$-bounded assuming the asymptotic nonlinearity of $\beta$.

Proof of Theorem 3: By Theorem 1, the generalized minimizers $\hat{g}_{\mathcal{C}_{n}}$ exist. For $n \geqslant m$ it follows from ineq. (1) and $\mathcal{C}_{m} \subseteq \mathcal{C}_{n}$ that

$$
H_{\beta}(g) \geqslant J_{\beta}^{[n]}+B_{\beta}\left(g, \hat{g}_{\mathcal{C}_{n}}\right), \quad g \in \mathcal{C}_{m} .
$$

Since the left-hand side of (7) can be finite the sequence $\hat{g}_{\mathcal{C}_{n}}$ is reversely $\beta$-bounded. Then, it is bounded locally in measure by Corollary 5 , using that $\beta$ is asymptotically nonlinear.

For a minimizing sequence $g_{k}$ in $\mathcal{C}_{m}$, Theorem 1 implies $g_{k} \rightsquigarrow \hat{g}_{\mathcal{C}_{m}}$, and then a subsequence converges $\mu$-a.e. Limiting in (7) along the subsequence

$$
J_{\beta}^{[m]} \geqslant J_{\beta}^{[n]}+B_{\beta}\left(\hat{g}_{\mathcal{C}_{m}}, \hat{g}_{\mathcal{C}_{n}}\right), \quad n \geqslant m
$$

on account of the lower semicontinuity of Bregman distance [5, Lemma 2.12]. Since the sequence $J_{\beta}^{[n]}$ is bounded and monotone $B_{\beta}\left(\hat{g}_{\mathcal{C}_{n}}, \hat{g}_{\mathcal{C}_{m}}\right) \rightarrow 0, n \geqslant m \rightarrow \infty$. By Lemma 4, there exists a function $h^{\infty}$ such that $\hat{g}_{\mathcal{C}_{n}} \rightsquigarrow h^{\infty}$.

Limiting in (7) with $n \rightarrow \infty$, along a subsequence,

$$
H_{\beta}(g) \geqslant \lim _{n \rightarrow \infty} J_{\beta}^{[n]}+B_{\beta}\left(g, h^{\infty}\right), \quad g \in \mathcal{C}_{m} .
$$

analogously as above. Since $m \geqslant 1$ is arbitrary this inequality holds even for $g \in \mathcal{C}_{\infty}$. Then, $\inf _{\mathcal{C}_{\infty}} H_{\beta}$ is at least the finite limit, and $J_{\beta}^{[\infty]}=\lim _{n \rightarrow \infty} J_{\beta}^{[n]}$ by monotonicity. Hence,

$$
H_{\beta}(g) \geqslant J_{\beta}^{[\infty]}+B_{\beta}\left(g, h^{\infty}\right), \quad g \in \mathcal{C}_{\infty},
$$

and the minimizing sequences in $\mathcal{C}_{\infty}$ are $\beta$-bounded. Theorem 1 applies to $\mathcal{C}_{\infty}$ and, by uniqueness, $h^{\infty}=\hat{g}_{C_{\infty}}$.

Limiting in (8) with $n \rightarrow \infty$, along a subsequence that gives $\hat{g}_{\mathcal{C}_{n}} \rightarrow \hat{g}_{C_{\infty}}, \mu$-a.e.,

$$
J_{\beta}^{[m]} \geqslant \lim _{n \rightarrow \infty} J_{\beta}^{[\infty]}+B_{\beta}\left(\hat{g}_{\mathcal{C}_{m}}, \hat{g}_{C_{\infty}}\right), \quad m \geqslant 1 .
$$

Then, $B_{\beta}\left(\hat{g}_{\mathcal{C}_{m}}, \hat{g}_{C_{\infty}}\right) \rightarrow 0$ when $m \rightarrow \infty$.

\section{ACKNOWLEDGEMENT}

The work on this contribution was supported by the Hungarian National Foundation for Scientific Research under Grant K 105840 and by Grant Agency of the Czech Republic under Grant 16-12010S.

\section{REFERENCES}

[1] Borwein, J.M. and Lewis, A.S., Convergence of best entropy estimates. SIAM J. Optimization 1 (1991) 191-205.

[2] Borwein, J.M. and Lewis, A.S., Strong rotundity and optimization. SIAM J. Optimization 4 (1994) 146-158.

[3] Csiszár, I., Generalized projections for non-negative functions. Acta Math. Hungar. 68 (1995), 1-2, 161-185.

[4] Csiszár, I. and Matúš, F., Information projections revisited. IEEE Trans. Inform. Theory 49 (2003) 1474-1490.

[5] Csiszár, I. and Matúš, F., Generalized minimizers of convex integral functionals, Bregman distance, Pythagorean identities. Kybernetika 47 (2012) 637-689.

[6] Harremoës, P. and Holst, K.K., Convergence of Markov chains in information divergence. J. Theoretical Probability 22 (2009) 186-202. Press, Princeton 1970.

[7] Rockafellar, R.T. and Wets, R.J-B., Variational Analysis. Springer Verlag, Berlin, Heidelberg, New York 2004.

[8] Teboulle, M. and Vajda, I., Convergence of best $\varphi$-entropy estimates. IEEE Transactions on Information Theory 39 (1993) 297-301. 
PROOFS OF THE RESUlTS FROM SECTION IV

This is an appendix which is not a part of the publication. It provides supplementary material to Section IV.

Proof of Lemma 1: Let $\nu$ denote the measure with the $\mu$-density $h$. Assuming the $\gamma$-divergence is finite,

$$
\begin{aligned}
D_{\gamma}(g, h) & =\int_{Z} h \gamma\left(\frac{g}{h}\right) d \mu=\int_{Z} \gamma\left(\frac{g}{h}\right) d \nu=\int_{Z} \gamma\left(\frac{g}{h}\right)_{\mid \mathcal{Y}, \nu} d \nu \\
& =\int_{Z} \gamma\left(\frac{g}{h}\right)_{\mid \mathcal{Y}, \nu} h d \mu=\int_{Z} \gamma\left(\frac{g}{h}\right)_{\mid \mathcal{Y}, \nu} h_{\mid \mathcal{Y}, \mu} d \mu .
\end{aligned}
$$

Conditional Jensen's inequality with $\gamma \geqslant 0$ implies

$$
\gamma\left(\frac{g}{h}\right)_{\mid \mathcal{Y}, \nu} \geqslant \gamma\left(\left(\frac{g}{h}\right)_{\mid \mathcal{Y}, \nu}\right) \text {. }
$$

By assumptions, $f_{\mid \mathcal{Y}}$ and $h_{\mid \mathcal{Y}}$ are well-defined and so is $g_{\mid \mathcal{Y}}$ for $g \in \mathcal{D}_{\mathcal{Y}, f}$. For such $g$ and $Y \in \mathcal{Y}$

$$
\begin{aligned}
\int_{Y} g_{\mid \mathcal{Y}, \mu} d \mu & =\int_{Y} g d \mu=\int_{Y} \frac{g}{h} d \nu \\
& =\int_{Y}\left(\frac{g}{h}\right)_{\mid \mathcal{Y}, \nu} d \nu=\int_{Y}\left(\frac{g}{h}\right)_{\mid \mathcal{Y}, \nu} h d \mu \\
& =\int_{Y}\left(\left(\frac{g}{h}\right)_{\mid \mathcal{Y}, \nu} h\right)_{\mid \mathcal{Y}, \mu} d \mu=\int_{Y}\left(\frac{g}{h}\right)_{\mid \mathcal{Y}, \nu} h_{\mid \mathcal{Y}, \mu} d \mu
\end{aligned}
$$

Using that $h_{\mid \mathcal{Y}, \mu}>0$,

$$
\left(\frac{g}{h}\right)_{\mid \mathcal{Y}, \nu}=\frac{g_{\mid \mathcal{Y}, \mu}}{h_{\mid \mathcal{Y}, \mu}} .
$$

It follows by $g_{\mid \mathcal{Y}, \mu}=f_{\mid \mathcal{Y}, \mu}$ that

$$
D_{\gamma}(g, h) \geqslant \int_{Z} \gamma\left(\frac{g_{\mid \mathcal{Y}, \mu}}{h_{\mid \mathcal{Y}, \mu}}\right) h_{\mid \mathcal{Y}, \mu} d \mu=D_{\gamma}\left(f_{\mid \mathcal{Y}, \mu}, h_{\mid \mathcal{Y}, \mu}\right) .
$$

Thus, the minimum is at least $D_{\gamma}\left(f_{\mid \mathcal{Y}}, h_{\mid \mathcal{Y}}\right)$.

Denoting $f_{\mid \mathcal{Y}} h / h_{\mid \mathcal{Y}}$ by $f^{h}$, for $Y \in \mathcal{Y}$

$$
\int_{Y} f d \mu=\int_{Y} f_{\mid \mathcal{Y}} d \mu=\int_{Y}\left(f^{h}\right)_{\mid \mathcal{Y}} d \mu=\int_{Y} f^{h} d \mu
$$

whenever the integral on the left is finite. Therefore, $f^{h}$ belongs to $\mathcal{D}_{\mathcal{Y}, f}$. As $D_{\gamma}\left(f^{h}, h\right)=D_{\gamma}\left(f_{\mid \mathcal{Y}}, h_{\mid \mathcal{Y}}\right)$ the minimum equals $D_{\gamma}\left(f_{\mid \mathcal{Y}}, h_{\mid \mathcal{Y}}\right)$ and $f^{h}$ is a minimizer.
Proof of Lemma 2: By Lemma 1, $D_{\gamma}\left(f_{\mid \mathcal{Y}_{n}}, h_{\mid \mathcal{Y}_{n}}\right) \nearrow$ and the limit of the sequence is at most $D_{\gamma}\left(f_{\mid \mathcal{Y}_{\infty}}, h_{\mid \mathcal{Y}_{\infty}}\right)$. A martingale theorem implies $\mu$-a.e. convergences $f_{\mid \mathcal{Y}_{n}} \rightarrow f_{\mid \mathcal{Y}_{\infty}}$ and $h_{\mid \mathcal{Y}_{n}} \rightarrow h_{\mid \mathcal{Y}_{\infty}}$. Hence,

$$
\liminf _{n \rightarrow \infty} D_{\gamma}\left(f_{\mid \mathcal{Y}_{n}}, h_{\mid \mathcal{Y}_{n}}\right) \geqslant D_{\gamma}\left(f_{\mid \mathcal{Y}_{\infty}}, h_{\mid \mathcal{Y}_{\infty}}\right)
$$

by Fatou lemma and lower semicontinuity of $\gamma \geqslant 0$.

Proof of Corollary 2: By monotonicity of $\mathcal{Y}_{n}, \mathcal{D}_{\mathcal{Y}_{n}, f} \searrow$. A function $g$ belongs to the intersection of $\mathcal{D}_{\mathcal{Y}_{n}, f}$ if and only if $\int_{Y} g d \mu=\int_{Y} f d \mu$ for $Y \in \bigcup_{n \geqslant 1} \mathcal{Y}_{n}$. The equality holds also for $Y \in \mathcal{Y}_{\infty}$. Hence, $\mathcal{C}_{n}=\mathcal{D}_{\mathcal{Y}_{n}, f}$ intersect to $\mathcal{C}_{\infty}=\mathcal{D}_{\mathcal{Y}_{\infty}, f}$. If $H_{\gamma}\left(f_{\mid \mathcal{Y}_{\infty}}\right)=D_{\gamma}\left(f_{\mid \mathcal{Y}_{\infty}}, 1\right)$ is finite then, by Lemma 1 with $h \equiv 1, \hat{g}_{\mathcal{C}_{n}}=f_{\mid \mathcal{Y}_{n}}$ and $\hat{g}_{\mathcal{C}_{\infty}}=f_{\mid \mathcal{Y}_{\infty}}$. Lemma 2 and Theorem 2 with $h_{\infty}=\hat{g}_{\mathcal{C}_{\infty}}$ imply the convergence.

Proof of Corollary 3: The integrand $\beta$ is asymptotically nonlinear and $\mathcal{C}_{n}=\mathcal{D}_{\mathcal{Y}_{n}, f} \nearrow$ and have the union $\mathcal{C}_{\infty}$ contained in $\mathcal{D}_{\mathcal{Y}_{\infty}, f} .^{\ddagger}$ Lemma 1 implies that $J_{\beta}^{[n]}=D_{\gamma}\left(f_{\mid \mathcal{Y}_{n}}, h_{\mid \mathcal{Y}_{n}}\right) \searrow$. Then, the sequence is finite and has a nonnegative limit because $\gamma \geqslant 0$. It follows by Theorem 3 that $B_{\beta}\left(\hat{g}_{\mathcal{C}_{n}}, \hat{g}_{C_{\infty}}\right) \rightarrow 0$ where $\hat{g}_{\mathcal{C}_{n}}=f_{\mid \mathcal{Y}_{n}} h / h_{\mid \mathcal{Y}_{n}}$. Then, by Fatou lemma

$$
\begin{aligned}
0 \geqslant \int_{Z} \liminf _{n \rightarrow \infty}\left[h \gamma\left(\frac{f_{\mid \mathcal{Y}_{n}}}{h_{\mid \mathcal{Y}_{n}}}\right)-h \gamma\left(\frac{\hat{g}_{C_{\infty}}}{h}\right)\right. \\
\left.-\gamma^{\prime}\left(\frac{\hat{g}_{C_{\infty}}}{h}\right)\left[\frac{f_{\mid \mathcal{Y}_{n}} h}{h_{\mid \mathcal{Y}_{n}}}-\hat{g}_{C_{\infty}}\right]\right] d \mu \geqslant 0 .
\end{aligned}
$$

In turn, $B_{\beta}\left(f_{\mid \mathcal{Y}_{\infty}} h / h_{\mid \mathcal{Y}_{\infty}}, \hat{g}_{C_{\infty}}\right)=0$, using that $f_{\mid \mathcal{Y}_{n}} \rightarrow f_{\mid \mathcal{Y}_{\infty}}$ and $h_{\mid \mathcal{Y}_{n}} \rightarrow h_{\mid \mathcal{Y}_{\infty}}$, $\mu$-a.e. Hence, $\hat{g}_{C_{\infty}}=f_{\mid \mathcal{Y}_{\infty}} h / h_{\mid \mathcal{Y}_{\infty}}$ and

$$
B_{\beta}\left(\hat{g}_{\mathcal{C}_{n}}, \hat{g}_{C_{\infty}}\right)=D_{\gamma}\left(f_{\mid \mathcal{Y}_{n}}, h_{\mid \mathcal{Y}_{n}}\right)-D_{\gamma}\left(f_{\mid \mathcal{Y}_{\infty}}, h_{\mid \mathcal{Y}_{\infty}}\right) \rightarrow 0 .
$$

This rewrites to $B_{\gamma}\left(f_{\mid \mathcal{Y}_{n}}, f_{\mid \mathcal{Y}_{\infty}}\right)=H_{\gamma}\left(f_{\mid \mathcal{Y}_{n}}\right)-H_{\gamma}\left(f_{\mid \mathcal{Y}_{\infty}}\right) \rightarrow 0$ when $h \equiv 1$. 